\title{
ÜBER DIE SCHWIERIGKEIT, DEM TÜRKISCHEN NATIONALDISKURS ZU ENTKOMMEN: ALEVITEN IN DEUTSCHLAND UND „HÜRRIYET“
}

\section{Martin Sökefeld}

\section{Einleitung: Nation und Differenz}

Dieser Band handelt von Migration und vom Paradigma kultureller Differenz. Wir diskutieren in den verschiedenen Beiträgen die These, dass kulturelle Differenz nichts ist, was Einwanderern und ihren Nachkommen durch die Migrationsbewegung unauflöslich zu kommt und sie daher dauerhaft - womöglich für immer - zu ,Fremden" in Deutschland macht. Auch solche Migranten oder Post-Migranten, die in der deutschen Gesellschaft ohne Schwicrigkeiten zurecht kommen, die etwa das hiesige Bildungssystem durchlaufen und beruflichen Erfolg haben, werden in Deutschland als „Fremde" und „Ausländer" kategorisiert - selbst wenn sie die deutsche Staatsangehörigkeit besitzen. So liegt der Schluss nahe, dass kulturelle Fremdheit nichts ist, was im Wesen der Einwanderer liegt, sondern dass sie etwas ist, was ihnen in gesellschaftlichen Diskursen und Praktiken immer wieder zugeschrieben wird. Verstärkt wird diese Zuschreibung dadurch, dass ganz überwiegend nur diejenigen Migranten wahrgenommen und diskutiert werden, die anscheinend Schwicrigkeiten haben und „Probleme machen", nicht aber die, die - in welcher Hinsicht auch immer - „erfolgreich" sind.'

1 Ausgenommen sind hicr dic wenigen ..Vorzeigetürken“, dic ständig als Bcispiele angeführt werden, wie etwa der Reiseunternchmer Vural Öger oder der ehemalige Bundestagsabgeordnete Cem Özdemir. Die Art und Wcise, wie diese Personen in der Öffentlichkeit präsentiert werden, verstärkt jedoch das Bild, dass die Einwanderer aus der Türkei insgesamt eine Problemgruppe sind. Sie werden nämlich in der Regel als Kontrast zur Mchrheit der Einwandercr dargestelit. Während auf diese Weise gesellschaftlicher und beruflicher Erfolg individualisiert wird, wird gesellschaftliches Scheitern „sozialisiert,“ denn wenn es um die Diskussion etwa von "Ausländerkriminalităt" geht, werden Probleme gerade nicht einzelnen Personen sondern der Kategoric "Ausländer“ insgesamt zugeschrieben. 
Man kann Ursachenforschung betreiben und fragen, warum Einwanderer dauerhaft kulturell ausgegrenzt und zu Fremden erklärt werden. Man wird dann zu der Erkenntnis kommen, dass die Abgrenzung von ,anderen“ ein wesentliches Merkmal der Konstruktion nationaler Identität ist (Wimmer 2002, vgl. Lanz in diesem Band). Seit Herders Ideen, die gegen Ende des 18. Jahrhunderts formuliert wurden, besteht im deutschen Nationsverständnis ein enger Zusammenhang von Nation und Kultur: Kultur definiert die Nation und grenzt sie gegenüber anderen Nationen ab. „Deutsche Kultur" wird durch die Abgrenzung von ,anderen Kulturen" definiert und nicht durch etwas, was ihr selbst zu Eigen wäre. Das wurde deutlich in der Diskussion um das Konzept der "Leitkultur", in der klare Vorstellungen davon zum Ausdruck kamen, was nicht zur deutschen Kultur gehört, die aber letztlich daran scheiterte, zu bestimmen, was eigentlich deutsch sei.

Die deutsche Nation wird als „Kulturnation" begriffen. Herder verstand Kultur als etwas, das Menschengruppen voneinander unterscheidet, das von Generation zu Generation weiter gegeben wird und Verschiedenartigkeit dauerhaft aufrecht erhält. In Deutschland liegt diese Idee einem genetischen Nationsverständnis zugrunde: Angehöriger der Nation wird man nicht (wie im politischen, partizipatorischen Nationsverständnis Frankreichs ${ }^{2}$ ) durch Teilhabe an der politischen Gemeinschaft, sondern durch Abstammung. Wenn man nicht die korrekte Abstammung vorweisen kann, bleibt man von politischer Partizipation ausgeschlossen. Erst die Reform des Staatsangehörigkeitsrechts hat begonnen, ein neues Nationsverständnis in Deutschland zu institutionalisieren. Nicht zuletzt deswegen war das neue Gesetz heftig umstritten. Die Idee der Kulturnation fußt auf der Vorstellung eines kulturell homogenen Staatsvolks, und diskursiv wird dieses Volk „rein“" gehalten, egal wie heterogen die Bevölkerung tatsächlich ist. Im Diskurs der Kulturnation wird Differenz nach außen verlegt: Differenz kennzeichnet nicht die Angehörigen der Nation untereinander sondern markiert die Grenze zu anderen Nationen.

Diese Ideen und Konzepte liegen zugrunde, wenn zwar einerseits die Integration von "Ausländern" in Deutschland gefordert wird, wenn in diesen "Integrationsdiskursen" Einwanderer aber immer als die kulturell Fremden, Anderen definiert werden, so dass ,Integration" schon rhetorisch verhindert wird. Ein Recht auf vollständige Teilhabe in Deutschland hat danach eben nur, wer schon "deutsch" ist.

\section{Nation und Emigration in der Türke}

Die Definition der Einwanderer aus der Türkei in Deutschland als Andere und Fremde im Land wird jedoch nicht nur von deutschen Diskursen vorgenommen, sondern auch vom Nationaldiskurs in der Türkei. Ähnlich wie Deutsch-

2 Siehe Brubaker (1992) furr die unterschiedlichen Konzeptionen der Nation in Frankreich und Deutschland. land ist die Türkei eine „späte Nation“: Eine türkische Nationalideologie und ein türkischer Nationalstaat entstanden crst, als der Nationalstaatsgedanke in den ehemals vom osmanischen Reich beherrschten nicht-türkischen Gebicten, etwa in Griechenland, schon sehr weit fortgeschritten war. Die türkische Nationalideologie, die von den Jungtürken und später vor allem vom Mustafa Kemal vertreten und umgesetzt wurde, versuchte im übrig gebliebenen, überwiegend türkischen Rest des osmanischen Reiches zu retten, was noch zu retten war. Zur Ausformulierung einer Ideologie, die eine türkische Nation postulierte bzw. ihr Wesen genauer explizierte, kam es sogar zum Teil erst nach Gründung des türkischen Nationalstaats. ${ }^{3}$ Umso massiver wurde dann die Idee einer homogenen türkischen Nation mit Jahrtausende alten geschichtlichen Wurzeln vertreten. Die kemalistische Nationalideologie ist bei allem Wande in der Türkei auch heute immer noch sehr wirkmächtig. Kulturelle Heterogein der Türkei auch heute immer noch sehr wirkmächtig. Kulturelle Heteroge-
nität wird - in Bezug auf Minderheiten wie Kurden oder Aleviten - in der Türkei immer noch weitgehend negiert. Damit beschäftige ich mich in diesem Beitrag aber nicht. Mir geht es statt dessen um die Ausdehnung des türkischen Nationaldiskurses auf Migranten aus der Türkei, die im Ausland leben, in unserem Fall in Deutschland. Ich möchte am Beispiel der aus der Türkei eingewanderten Aleviten zeigen, dass dieser Diskurs die dauerhafte Definition der Emigranten als Türken fortschreibt.

Nicht nur staatliche Institutionen, die unter dem Einfluss oder der Kontrolle der türkischen Konsulate stehen, arbeiten daran, einen solchen nationalkulturellen Konsens in das Leben der Migranten aus der Türkei in Deutschland einzuschreiben. Vielleicht noch wichtiger und wirksamer sind in dieser Hinsicht einige der in Deutschland vertriebenen türkischen Massenmedien. Benedict Anderson (1983) hat in seiner Studie über die „Erfindung der Nation" eindrücklich auf die Bedeutung von Printmedien für die Entwicklung nationalistischer Ideologien hingewiesen. Ebenso wichtig sind intemational konsumierbare Massenmedien heute dafur, den "long distance nationalism" (Anderson 1998) in Diasporagemeinschaften oder unter Emigranten zu konstituieren und aufrechtzuerhalten.

Die türkische „mediascape“ (Appadurai 1989) in Deutschland ist schr umfangreich. Nachdem sich seit den 1970er Jahren türkische Presseorgane in Deutschland etablierten, zum Teil mit eigenen Europaausgaben, kamen nach der Privatisierung des türkischen TV-Marktes Anfang der 1990er Jahre zahlreiche in Deutschland empfangbare Fernschsender hinzu. ${ }^{4}$ Studien, die sich mit der Rolle türkischer Medien für Einwanderer aus der Türkei in Deutsch-

3 Ich beziehe mich hier auf die ,türkische Geschichtsthese“ (Türk Tarih Tesi), die erst 1932 offiziell proklamiert wurde (vgl. Yörük 1997). Sie erklärt dic Türken zu den Schöpfern einer in Zentralasien entstandenen Ur-Zivilisation, aus der alle späteren Hochkulturen hervorgegangen sind und verschafft der türkischen $\mathrm{Na}$ tion damit die quasi zeitlose historische Tiefe, die so charakteristisch für nationalistische Geschichtskonstruktionen ist.

4 Zur Entwicklung des Privatfernsehens in der Türkei siche Aksoy und Robins 2000. 
land beschäftigt haben, sind dabei uberwiegend zu dem den kritischen Schluss gekommen, dass viele Publikationen der Integration von Einwanderern in die bundesdeutsche Gesellschaft eher entgegenwirken würden. ${ }^{5}$ Schon fast berüchtigt ist in diesem Zusammenhang die Tageszeitung Hürriyet. Hürriyet ist die türkische Zeitung mit der höchsten Auflage nicht nur in der Türkei selbst, sondern auch in Deutschland. In diesem Beitrag diskutiere ich eine Kampagne von Hürriyet, welche die Zeitung in den Jahren 2000 und 2001 gegen die "Föderation der Alevitengemeinden in Deutschland" (Almanya Alevi Birlikleri Federasyonu, AABF), den größten Dachverband alevitischer Einwanderer aus der Türkei in Deutschland, publizierte und die in dem Vorwurf gipfelte, die AABF würde die Türken in Deutschland ihrer Nationalität entfremden und sich dabei zum Handlanger der deutschen Regierung und der Europäischen Union machen.

Bevor ich auf diese Pressekampagne eingehe, werde ich zunächst kurz das Verhältnis zwischen Aleviten und dem Staat in der Türkei sowie die Stellung von Hïrriyet in der turkischen Presselandschaft in Deutschland darstellen.

\section{Aleviten und der türkische Staat:}

\section{Ein a mbivalentes Verhältnis}

Mir fehlt an dieser Stelle der Platz, um ausfuhrlich auf das Alevitentum eingehen zu können. ${ }^{6}$ Ich muss mich mit dem knappen Hinweis begnügen, dass Aleviten eine "heterodoxe" Gemeinschaft bilden, die sich auf den Imam Ali zurückführt und ihre spezifische Form im historischen Anatolien, u. a. im Machtkampf zwischen Osmanen und Safawiden, gefunden hat. Aleviten wurden im osmanischen Reich von staatlichen Einrichtungen und der sunnitischen Mehrheitsbevölkerung marginalisiert. Es gab zahlreiche Gutachten (fetwas) sunnitischer Rechtsgelehrter, in denen Aleviten als Apostaten und damit Vogelfreie bezeichnet wurden. Infolgedessen wurde die alevitische Religion fast ausschließlich im Geheimen praktiziert. Es gibt sowohl kurdischsprachige als auch türkischsprachige Aleviten.

War das Verhältnis zwischen osmanischem Staat und alevitischer Bevölkerung eindeutig negativ geprägt, so ist das Verhältnis zwischen Aleviten und der türkischen Republik ambivalent. Einerseits setzten Aleviten große Hoffnungen auf die Gründung der Republik und in die kemalistischen Reformen, andererseits brachte die Republik nicht im vollen Umfang das, was sich die

5 Am Ende ihrer Untersuchung uber türkische Medien in Deutschland kommen Cryns et al. (1991: 78) zum folgenden Schluss: "Integration im Sinne einer Synthese verschiedener Kulturen oder einer wechselseitigen Fortentwicklung scheint für die türkischen Zeitungen also indiskutabel." Neuere Untersuchungen relativieren diesen Befund, indem sie darauf hinweisen, dass die Mehrheit der Migranten keineswegs nur to

6 Für ausfuhrliche Darstellungen siehe Kehl-Bodrogi 1988, Dressler 2002 und White/Jongerden 2003.
Aleviten von ihr versprachen. Der Laizismusgedanke, die Abschaffung der Scharia und die Betonung der Gleichheit der Geschlechter, um nur einige Elemente des republikanischen Gedankenguts herauszugreifen, wurde von Aleviten einhellig begrüß, ganz im Gegensatz zur konservativen sunnitischen Geistlichkeit. Atatürks Reformen implizierten eine gesellschaftliche Umorientierung kaum nachvollziehbaren Ausmaßes, die bei weitem nicht von der gesamten Bevölkerung getragen wurde. Umso wichtiger war es für Atatürk, sich der Unterstützung solcher Bevölkerungsgruppen zu versichern, die mit seinen Vorstellungen sympathisierten. Und dazu gehörten auch die Aleviten. Es gab im November 1919, nach den Kongressen von Erzurum und Sivas, die Atatürks Aufstieg zu einem ,nationalen" Führer markierten, ein gehcimnisumwittertes Treffen zwischen Atatürk und Cemalettin Çelebi, dem Vorsteher des Bektaşi-Ordens, der als der oberste Repräsentant der Aleviten angesehen wurde. Bei diesem Treffen sicherte Cemalettin Celebi Atatürk die Unterstützung durch die Aleviten zu (Dressler 1999: 94ff). Tatsächlich gehörten in den folgenden Jahrzehnten Aleviten zu den Stammwählern der von Atatürk begründeten Cumhuriyet Halk Partisi (CHP, Republikanische Volkspartei). ${ }^{7}$

Andererseits hatte die Gründung der türkischen Republik keine Religionsfreiheit für die Aleviten zur Folge. Im Jahr 1925 wurde ein Gesetz verabschiedet, das alle "heterodoxen" Institutionen und Praktiken, einschlicßlich der alevitischen, unter Strafe stellte. Auch die Negation der kulturellen Heterogenität der Bevölkerung in der Türkei betraf die Aleviten. So verbietet das türkische Vereinsrecht bis heute die Gründung von Organisationen auf der Basis irgendeiner Partikularidentität, weil damit die Einheit der türkischen Nation in Frage gestellt werde und somit der Tatbestand des Separatismus gegeben sei. Die Gründung explizit alevitischer Vereinigungen wird in der Türkei mit Verweis auf diese Bestimmungen daher häufig gerichtlich verboten.

$\mathrm{Zu}$ einem Machtfaktor wurde die Opposition von Aleviten gegen den türkischen Staat vor allem in den 1970er Jahren, als besonders junge Aleviten sich den verschiedenen linksrevolutionären Organisationen anschlossen und an der Polarisierung der türkischen Gesellschaft zwischen ,rechts" und "links" wichtigen Anteil hatten. „Alevitisch" wurde damals ebenso mit „links" gleichgesetzt wie „sunnitisch“ mit „rechts“. In den Jahren 1978 bis 1980 kam es in verschiedenen Städten zu gewalttätigen Auseinandersetzungen zwischen Rechten und Linken, denen zahlreiche Aleviten zum Opfer fielen. Aleviten sprechen hier stets von „Massakern" und werfen den staatlichen Organen vor, damals nicht nur Aleviten nicht geschützt zu haben, sondern zum Teil auf der Seite von Nationalisten und Faschisten gegen Aleviten vorgegangen zu scin.

In der Folge dieser politischen Entwicklung kamen zahlreiche Aleviten als politische Flüchtlinge nach Deutschland, die hicr ihre Oppositionspolitik gegen den türkischen Staat fortsetzten. Als sich dann scit dem Ende der 1980er Jahre eine organisierte alevitische Szene in Deutschland zu formieren begann, befanden sich unter den Führungspersönlichkeiten dieser neuen alevitischen

7 Vgl. Schüler 1998. 
Bewegung zahlreiche ehemalige Aktivisten linker Exilorganisationen. ${ }^{8}$ Sie hatten zwar ihre revolutionären Bestrebungen aufgegeben, standen aber dennoch dem türkischen Staat distanziert gegenüber und weigerten sich etwa, mit den türkischen Konsulaten zusammenzuarbeiten. In der ersten Hälfte der 1990er Jahre gab es weitere Gewaltereignisse in der Türkei gegen Aleviten, was dazu führte, dass die Distanz zum türkischen Staat eher noch verstärkt wurde. So ziehen Aleviten in Deutschland häufig einen Vergleich zwischen Deutschland und der Türkei und betonen, dass sie in Deutschland eine Freiheit genießen, die ihnen im ,eigenen Land“ (d.h. in der Türkei) vorenthalten wird.

Allerdings stellen sich nicht alle Aleviten in Deutschland (und schon gar nicht alle in der Türkei) eindeutig in Opposition zum türkischen Staat. Viele der Migranten verstehen sich nach wie vor als Kemalisten und kritisieren die ablehnende, oppositionelle Haltung der anderen. Oft kommt es darüber in alevitischen Organisationen zum Konflikt - ein Konflikt, den die Zeitung Hürriyet für ihre $Z$ wecke zu instrumentalisieren versuchte.

\section{Hürriyet in Deutschland}

Hürriyet ist die meistgelesene türkische Tageszeitung in Deutschland. ${ }^{9} 1948$ wurde das Blatt in der Türkei gegründet, seit Anfang der 1970er Jahre ist es auch in Deutschland erhältlich und dominiert eindeutig den türkischsprachigen Pressemarkt im Land. Einer Umfrage jüngeren Datums zu folge ist für 38 Prozent der Einwanderer aus der Türkei Hürriyet die bevorzugte Tageszeitung. ${ }^{10}$ Hürriyet vertritt eine strikt nationalistisch-kemalistische Position, die sich schon in der Gestaltung des Titelblatts ausdrückt. Im Kopf der Titelseite befindet sich neben dem Titel „Hürriyet“ (Freiheit) eine türkische Flagge, die über einer Silhouette Atatürks weht, unterstrichen von dem Satz: „Türkiye Türklerindir" - „Die Türkei gehört den Türken." Karacabey (1996) zu Folge beabsichtigt Hürriyet eindeutig, die Meinung der Migranten aus der Türkei in Deutschland zu beeinflussen. In Deutschland wird eine Europaausgabe der Zeitung produziert und vertrieben, die deutschlandweit sowie regional und lokal orientierte Seiten umfasst. Die Berichterstattung über Ereignisse in Deutschland folgt ebenfalls der nationalistischen Perspektive. In der Regel wird nur über solche Ereignisse berichtet, welche die Migranten unmittelbar betreffen. Dabei spendet Hürriyet stets solchen Aktivitäten Beifall, welche die "türkische Identităt" der Migranten fördern und ist sehr kritisch in Bezug auf Integration eingestellt, sofern damit die "Gefahr" der Entwicklung neuer, hy-

8. Zur Entwicklungen alevitischer Organisationen in Deutschland siehe Sökefeld und Schwalgin 2000.

9 Im Jahr 1997 hatte Hürriyet eine Tagesauflage von 107.000. Zum Vergleich: Die nächstgrößte Tageszeitung, Türkiye, kam auf lediglich 40.000 Exemplare (Heinemann und Kamcili 2000: 121).

10 die tageszeitung, 18. 6. 2001. brider oder gar "deutscher" Identitäten der Einwanderer verbunden ist (vgl. Cryns u. a. 1991: 56).

Hürriyet ist berüchtigt für einen Kampagnen-Journalismus, der auf Persönlichkeiten zielt, die nationalen türkischen Interessen - so, wie sie von Hürriyet verstanden werden - zuwider handeln. Häufig wurde der ehemalige grüne Bundestagsabgeordnete Cem Özdemir zum Opfer von Hïrriyets Berichterstattung. Nachdem er von Hürriyet zunächst als ,unser Mann im deutschen Parlament" gefeiert worden war, kühlte dic Begeisterung für Özdemir merklich ab, als er nicht als türkischer Interessenvertreter auftrat. Das Verhältnis schlug in offene Feindschaft um, weil Özdemir nicht mit Kritik z.B. an der Menschenrechtspolitik der türkischen Regierung sparte. Zuletzt (im Jahr 2001) wurde Özdemir von Hürriyet vorgeworfen, er habe einem armenischen Priester die Hand geküsst und sich damit einem Feind der türkischen Republik angedient. Inzwischen hat Özdemir in dieser Angelegenheit einen Prozess gegen Hürriyet gewonnen. Federfuhrend bei diesem Kampagnen-Journalismus, der sich nicht nur gegen Cem Özdemir richtete, war der Chef der EuropaAusgabe von Hürriyet, der auch schon als der „Karl-Eduard von Schnitzler der türkischen Presse" bezeichnet wurde (Ceyhun 1999: 7).

\section{Die Kampagne gegen die Föderation der}

Alevitengemeinden in Deutschland

Die türkische Presse in Deutschland berichtet auch immer wieder über dic Aktivitäten der alevitischen Organisationen. So schrieben Milliyet, Türkiye Özgür Politika und Hürriyet auch über die Generalversammlung der AABF, die Ende November 2000 stattfand, und auf der die Delegierten der alevitischen Vereine einen neuen Vorstand der Föderation wählten. Während sich die drei zuerst genannten Zeitungen mit Berichten über die Versammlung und Kommentaren zum Wahlergebnis begnügten, griff Hürriyet in die Ereignisse ein, indem die Zeitung Interna aus einem Finanzbericht der Föderation veröffentlichte, auf deren Basis Vorwürfe über finanzielle Unregelmäßigkeiten an die Adresse des Vorstands und vor allem des Vorsitzenden Turgut Öker gerichtet wurden. Hintergrund dieser Indiskretion war ein Konflikt im Vorstand der Föderation einige Monate zuvor, bei dem ein Teil des Vorstands zurückgetreten war. Man vermutete, dass die Interna aus dem Kreis der zurückgetretenen Vorstandsmitglieder oder ihrer Unterstützer an Hürriyet weiter gegeben wurden.

Hürriyets Berichterstattung endete nicht mit der Versammlung oder der Diskussion ihrer Ergebnisse. Turgut Öker war erneut zum Vorsitzenden gewählt worden. In den folgenden drei Monaten wurde der Vorstand, und häufig der Vorsitzende persönlich, von Hürriyet in oft mehreren Beiträgen täglich aufs Schärfste angegriffen. Eine zweite Periode der Kampagne gegen den Vorstand der Föderation folgte von Mitte Mai bis Ende Juni 2001. 
Die Aufmerksamkeit, die Hürriyet der Föderation widmete, war in der Tat außergewöhnlich. Man berichtete nicht über weitere Ereignisse, sondern äuBerte schwerste Kritik an der Föderation. Das Rückgrat der Kampagne waren die täglichen Kolumnen von Ertuğ Karakullukçu, dem Chef der Europaausgabe. Wăhrend der ersten Periode widmete er der Föderation 45 Kolumnen, dazu kamen fast täglich weitere Artikel, sowie ab Mitte Dezember Leserbriefe von Aleviten, in denen Kritik an der Föderation geäußert wurde. Der Tenor war strikt negativ. Es gab, auch unter den Leserbriefen, nicht einen einzigen Beitrag, der die Föderation oder auch nur Teile ihrer Arbeit positiv beurteilt hätte. Am Anfang standen dabei Finanzpraktiken im Vordergrund, ${ }^{11}$ später ging es um die allgemeine Zielsetzung der Arbeit der Föderation. Ich beschäftige mich in diesem Beitrag mit diesem zweiten Komplex der Kritik. Darin wird der Föderation Separatismus vorgeworfen, aber auch, dass sie sich auf die Seite der Armenier geschlagen habe, dass sie Atatürk und die türkische Fahne nicht ehre, dass sie überhaupt ein Feind des türkischen Staates sei, dass sie die Jugend auf Abwege fuhre, sowie dass sie die Türken in Deutschland von ihrem "Türkentum" entfremden und zu Deutschen machen wolle. Der Subtext all dieser Vorwüfe implizierte, dass die Föderation Zielsetzungen und Aktivităten verkörpere, die gegen den türkischen Staat und die türkische Nation gerichtet seien.

\section{Separatismus!}

Verschiedene dieser Themen sprach Ertuğ Karakullukçu in seiner Kolumne vom 1. Dezember 2000 an. Zunächst wendet er sich dem Thema Atatürk, bayrak (Nationalflagge) und toprak (Erde, Boden, d.h. Nationalterritorium) zu:

„Da ist zum Beispiel der Streit uber Atatürk, die Flagge und das Territorium, der immer wieder auf die Agenda der alevitischen Gemeinschaft gesetzt wird. Wir wissen, dass die alevitische Gemeinschaft mit der Flagge und dem Territorium kein Problem hat. Die Fahne mit Mond und Stern ist die Flagge von uns allen, und die Turkei ist unser gemeinsames Vaterland. Aber wie sollen wir es verstehen, dass während dieses Kongresses [der alevitischen Foderation] es als negative Haltung kommentiert wurde, dass Hürriyet einmal gerechtfertigter Weise darauf hingewiesen hat, dass die Flagge bei einem alevitischen Festival nicht gezeigt wurde? Wir wissen, dass in den Häusem unserer alevitischen Landsleute die türkische Fahne und Atatirk nicht fehlen. Wir wissen, dass in der alevitischen Gemeinsche kein Problem mit Fahne und Territorium gibt." "12

Diese Passage bezieht sich darauf, dass es sowohl in der Föderation als auch in lokalen alevitischen Vereinen häufig Diskussionen darüber gab, ob bei Veranstaltungen oder überhaupt in den Vereinsräumen türkische Nationalsymbole wie Portraits von Atatürk und die türkische Nationalfahne aufge-

11 Die Vorwüre sind später vor Gericht entkräftet worden; Hürriyet wurde dazu verurteilt, einen Widerruf zu veröffentlichen.

12 Übersetzung aller Zitate durch den Autor. hängt werden sollten, wie es bei nicht-oppositionellen Organisationen türkischer Migranten allgemein üblich ist. Vor allem Aleviten "linker" Herkunft lehnten es ab, diese Symbole zu zeigen, während kemalistisch eingestellte Aleviten Fahne und Atatürk forderten. ${ }^{13}$ Inzwischen hat sich in der Föderation die Haltung durchgesetzt, dass Ataturk und die türkische Fahne nationalpolitische Symbole seien, die im alevitischen Kontext nichts zu suchen haben, da das Alevitentum zwischen verschiedenen Nationen keinen Unterschied mache. ${ }^{14}$ Im Programm der AABF ist aber explizit festgehalten, dass damit nicht diese Symbole an sich abgelehnt wurden und dass mit der Haltung der AABF keine anti-türkische Einstellung verbunden sei (AABF 1998: 12). In der Folge eines Kulturfestivals der Föderation, das 1996 im: Kölner Fußballstadion ohne Fahnen und Atatürk stattgefunden hatte, hatte Hürriyet schon einmal in einer längeren Serie von Artikeln der Föderation eine anti-türkische Haltung vorgeworfen.

Im obigen Zitat wird Hürriyets rhetorische Strategie deutlich: Man greift die Föderation, bzw. ihren Vorstand oder den Vorsitzenden Turgut Öker an, vermittelt aber gleichzeitig das Bild, dass die kritisierte Haltung eben nur in der Föderation zu finden sei, nicht aber in der "alevitischen Gemeinschaft insgesamt. Es wird impliziert, dass die Föderation nicht tatsächlich die Aleviten vertrete, dass im Gegenteil die „alevitische Gemeinschaft" die Föderation genauso kritisiere, wie Hürriyet das tut. „Aleviten an sich" sind also auch in Deutschland gute türkische Patrioten - nur die AABF und ihr Vorstand sind es nicht. Man muss dabei wissen, dass diese Passage und sämtliche Artikel der Kampagne für Aleviten in Deutschland geschrieben wurden (in der Türkeiausgabe von Hürriyet erschienen sie nicht), egal, ob sie einen türkischen Pass besitzen oder einen deutschen. In den verschiedenen Artikeln der Kampagne werden Aleviten in Deutschland stets als ,yurttaşlarımı" oder „vatandaşla rimiz" bezeichnet, also etwa als "unsere Landsleute" oder "unsere Kompatrioten". Wahrend alevitische Migranten in Deutschland damit fest in die türkische Nation eingeschlossen werden, wird die Föderation als Verein von Separatisten dargestellt.

Dies wird im nächsten Absatz des selben Textes deutlich, in dem die (temporäre) Zusammenarbeit der Föderation mit dem kurdisch-alevitischen Dachverband in Deutschland angesprochen wird, die damals noch „Föderation der Aleviten Kurdistans" (Federasyon Elewiyen Kurdistani) hieß. Karakullukçu schreibt: „Was bedeutet ,Föderation der Aleviten Kurdistans'? Gibt es ein Kurdistan in der Türkei? Betrachtet der Vorstand der Föderation eine solche Entwicklung als legal?" Die AABF wurde aufgefordert, diese Fragen zu beantworten. Und schließlich wurde noch „alevitische Identităt" selbst ange-

13 Dies geht so weit, dass einige alevitische dedes (Geistliche) sich weigern, den alevitischen Ritus cem zu leiten, wenn Atatürk und dic Flagge nicht aufgehängt werden.

14 Interessanterweise werfen vicle kemalistische Aleviten der Föderation vor, sie sei „politisch". Mit „politisch" ist dann aber immer "links" und „oppositionell“" gemeint. Für die Kemalisten ist die türkische Fahne kcin ,politisches" Symbol. 
sprochen: „Sie tun so, als gäbe es eine separate alevitische Identität innerhalb der türkischen Nation. In dieser Hinsicht bestehen sie auf Identität. Sie treiben das Konzept der ,alevitischen Identität ${ }^{\prime}$ ins Extrem und sprechen aus dem Bemühen heraus, eine separate ethnische Gemeinschaft zu schaffen. ".15

Die Alevitische Föderation wird kontinuierlich als Kollaborateur der Feinde der Türkei, vor allem der Kurden und der Armenier, dargestellt und ihr wird damit vorgeworfen, die "alevitische Gemeinschaft" in eine falsche Richtung zu lenken und sie von den nationalen Werten der Türkei entfremden $\mathrm{zu}$ wollen. Insgesamt bestand die Kampagne gegen die AABF aus etwa 150 Texten, Kolumnen, Kommentaren und Leserbriefen. Einige der Vorwürfe waren sehr schwerwiegend, manche Angriffe geradezu beleidigend. Dabei war die Kampagne insgesamt ziemlich langweilig, denn ständig wurden dieselben Vorwürfe wiederholt. Und sie war offensichtlich unverhältnismäBig, denn kein anderes Thema genoss zur selben Zeit in den Seiten von Hürriyet eine auch nur annähernd ähnliche publizistische Aufmerksamkeit - nicht einmal die Wirtschaftskrise in der Türkei.

Die Föderation ist keine mächtige und sehr große Organisation, sie organisiert etwa 20.000 Aleviten in Deutschland. Warum hat die Europaausgabe von Hürriyet der Föderation dann so viel Aufmerksamkeit gewidmet? Manchmal sah auch Hürriyet die Notwendigkeit, diese Form der Berichterstattung zu begründen. Es wurde dann argumentiert, dass Hürriyet lediglich der journalistischen Pflicht, die Leser zu informieren, nachkäme. Das ist allerdings nicht sehr uberzeugend, da "Information" nicht aus der dauernden Wiederholung derselben Geschichten besteht. „Information" hätte auch erfordert, daß Hürriyet den Lesern mitgeteilt hătte, wenn Vorwürfe an die $\mathrm{AABF}$ vor Gericht widerlegt wurden. Aber das geschah nie in auch nur annähernd ausgewogener Weise. Die Kampagne hat nur Sinn, wenn Hürriyet die Föderation tatsächlich als Gefahr wahrgenommen hat, als Gefahr fur die nationalen Interessen der Türkei. Warum?

Die ideologische Basis des türkischen Nationalstaats ist die Vorstellung einer geeinten und homogenen Nation. Jeder Türke hat an erster Stelle teil an dieser Nation und leitet von ihr seine grundlegendste Identität und Zugehörigkeit ab. Er oder sie mag Kurdisch sprechen oder Alevit sein, aber das darf das prinzipielles Turkentum (türklük) nicht in Frage stellen. Keine andere Identifizierung darf dieselbe oder gar größere Bedeutung haben. Ein großer Teil der Geschichte der Republik Turkei kann als die Geschichte einer Identitătspolitik geschrieben werden, in der der Staat mit seinen Institutionen alles unternahm, um die Bedeutung der türkischen nationalen Identität seinen Subjekten einzuprägen. Jeder Diskurs und jedes Handeln, das diesen absoluten Vorrang der nationalen Identităt stört, ist bölücülük - Separatismus. Die Gefahr des Separatismus, der Abspaltung von Bevölkerungsgruppen, droht aber nicht nur im Inland, etwa durch der Forderung nach einem unabhängigen Kurdistan. Die türkische Nation ist längst nicht mehr auf das nationale Territorium der Türkei

15 Hürriyet, 1. Dezember 2000. beschränkt, da in Folge der Auswanderung zahlreiche Türken in anderen Staaten leben. Der Vorrang der nationalen Identität ist durch Auswanderung nicht gefährdet, solange die Migranten sich als Emigranten begreifen, d. h., solange die Türkei ihr zentraler Bezugspunkt bleibt. Dieser zentrale Bezug zur Türkei war in den ersten Jahren der Auswanderung unproblematisch, war die Migration doch auch von den Migranten selbst ebenso wie von den Migrationsländern als temporäre Phase angesehen worden, die durch die Rückkehr in die Türkei beendet werden würde. Aber diese fraglose Voraussetzung der Türkeiorientierung und der zukünftigen Rückkehr gilt, wie wir wissen, für die meisten Migranten inzwischen nicht mehr.

Von der Prämisse der Rückkehr ging zum Beispiel der muttersprachliche Ergänzungsunterricht aus, furr den der türkische Staat Lehrer an deutsche Schulen entsandte. Später wurde eine Auslandsorganisation der türkischen Religionsbehörde gegründet, die in Deutschland die spezifisch nationaltürkische Version des Islam fördern sollte. Die Sprache im Türkischunterricht war nicht einfach ein Mittel zur Kommunikation, das die Schüler erwerben sollten, sondern sie war Ausweis der Zugehörigkeit zu einer Nation. ${ }^{16}$ Nicht umsonst begannen viele türkische Lehrer den Unterricht mit der türkischen Nationalhymne oder mit dem Satz Atatürks: „Wie glücklich sind die, die von sich sagen können, ,ich bin Türke.'“

Ein Bericht der (staatlichen) Religionsbehörde stellt fest:

„Die Zahl der Angestellten unserer Behörde, die in diesen Ländern [der Emigration] arbeiten, wächst von Tag zu Tag. Sie leisten religiöse, soziale und kulturelle Dienste für unsere Bürger und ihre Kinder. Auf diese Weise werden unsere Menschen, die in diesen Ländern leben, dabei unterstützt, sich nicht zu assimilieren und ihre wahre Identität und Kultur zu bewahren" (zitiert nach Shankland 1999: 31).

Im Sprachunterricht, in religiossen Auslandinstitutionen und in Teilen der türkischen Presse wird somit ein exterritorialer Nationaldiskurs gepflegt, an dem sich die AABF jedoch nicht beteiligt. Sie tritt fur politischen Wandel in der Türkei ein und meint damit nicht nur Gleichberechtigung der Aleviten sondern Demokratisierung überhaupt. Außerdem will sie die Bewahrung alevitischer Identität in Deutschland fördern - aber diese Identität wird nicht als türkische Identität verstanden. Dies drückte der Vorsitzende einer alevitische Genossenschaft, der fruher auch Vorstandsmitglied der Föderation war, bei der Delegiertenversammlung im November 2000 aus, als er sagte: „Es wird versucht, das Alevitentum in einer tirrkisch-alevitischen Synthese zu ersticken. Wir müssen eine Kultur der Freiheit im Alevitentum schaffen." “17

Die Föderation begreift das Alevitentum als eine transstaatliche Zugehörigkeit und betont häufig, dass Aleviten nicht nur in der Türkei leben, sondern

16 Die ideologische Aufladung der türkischen Sprache in türkischen Nationaldiskurs zeigte sich besonders deutlich im Versuch, die turkische Sprache, analog zur Türk tarih tesi, zur "Ursprache" zu erklären (vgl. Lewis 1984).

17 Zitiert in Özgür Politika, 27. November 2000. 
auch in Ländern wie Albanien, Bulgarien, und nun vor allem auch in Deutschland. Aus diesem Blickwinkel hat das Alevitentum nichts mit einer spezifischen nationalen Identität zu tun. Daher engagiert sich die Föderation im Unterschied zu manchen anderen Organisationen von Migranten aus der Türkei in Deutschland - auch nicht darin, die türkische Identität der Migranten zu „bewahren“. Die Weigerung, die türkische Fahne oder Bilder von Atatürk aufzuhängen, spricht für sich. Die Föderation stellt das Alevitentum stets als ein sehr offenes, tolerantes und modernes Ideensystem dar, das an universellen Werten orientiert ist und willens ist, verschiedene positive Einflüsse aus anderen Quellen zu integrieren. Manchmal wird sogar gesagt, dass als Folge der Integration von Aleviten in die Staaten Westeuropas aus dem anatolischen Alevitentum ein europäisches Alevitentum entstehe.

Diese Haltung drückt sich in zahlreichen sozialen und politischen Aktivitäten der Föderation in Deutschland aus. So unterstützt die Föderation die Einbürgerung der Migranten in Deutschland und führte mit Förderung des Bundesministeriums des Inneren ein Staatsbürgerschaftsprojekt durch, das uber die rechtlichen Möglichkeiten und Bedingungen der Einbürgerung informierte. Zwar wurden Aleviten in Deutschland nicht direkt zur Einbürgerung aufgefordert, aber die Option der Einbürgerung wurde eindeutig mit groBer Sympathie bedacht. Überhaupt betont die Föderation, dass sie sich nicht als "Ausländerorganisation" versteht. Sie fordert ihre Mitglieder auf, sich in der deutschen Gesellschaft zu engagieren und zugehörig zu fühlen. Dabei wird auch immer wieder herausgestelit, dass aufgrund der vom Alevitentum vorgegebenen Werte Aleviten keine Integrationsschwierigkeiten hätten und der deutschen Gesellschaft sehr gut kompatibel seien. Die Fodderation verlangt die Einfuhrung von alevitischem Religionsunterricht in deutschen Schulen und hat im Unterschied zu manchen Konzepten des islamischen Religionsunterrichts von Anfang an betont, dass dieser Unterricht auf Deutsch durchgefulhrt werden soll.

\section{Transnationale Identitätspolitik}

Man kann die Politik der alevitischen Föderation in der Tat als Subversion der Idee einer geeinten, homogenen und klar abgegrenzten türkischen Nation begreifen. Das Türkentum wird nicht als die Zugehörigkeit verstanden, die vor allem anderen steht. Obwohl sich die AABF nicht als türkische Organisation versteht, bezieht sich doch ein wichtiger Teil ihrer Arbeit auf die Türkei. Anstatt dabei aber die ,nationalen Interessen der Türkei“ vertreten, benutzt die Föderation ihre Position in Deutschland und Europa, um Lobbyarbeit für Wandel in der Türkei und damit eine Art transnationale Identitätspolitik in Bezug auf die Türkei zu betreiben. ${ }^{18}$ Gerade die Europäische Union ist fur die Türkei eine höchst ambivalente Angelegenheit. Einerseits wird die Aufnahme

$18 \mathrm{Vgl}$. Sökefeld 2003: 151ff. in die EU gefordert, andererseits wurden Forderungen der EU nach Veränderungen in der Türkei als Voraussetzungen für den Beitritt häufig als Einmischung in die inneren Angelegenheiten zurückgewiesen. Die alevitische Föderation in Deutschland hat sich dafür eingesetzt, dass die EU dabei auch eine Veränderung der rechtlichen Situation der Aleviten in der Türkei einfordert. Der EU-Bericht aus dem Jahr 2000 zu den Beitrittsvorbereitungen der Türkei sagt:

„Die offizielle Haltung gegenüber den Aleviten scheint unverändert. Klagen der Aleviten betreffen vor allem den obligatorischen Religionsunterricht in den Schulen und Schulbücher, welche die alevitische Identität nicht berücksichtigen, sowie die Tatsache, dass es finanzielle Unterstützung lediglich für den Bau sunnitischer Moscheen und religioser Stiftungen gibt" (Europäische Kommission 2000: 18).

Die alevitische Föderation befürwortet den EU-Beitritt der Türkei, verlangt jedoch, dass die Türkei dazu weitere Reformen durchführen muss. Für diese Position setzt sich die Föderation auch gegenüber der Regierung in Berlin ein. Hürriyet wirft der Föderation vor, mit ihrem transnationalen politischen Engagement für die Demokratisierung in der Türkei und vor allem für die Gleichberechtigung der Aleviten gegen die Interessen der Türkei zu verstoßen (die z.B. in der bedingungslosen Aufnahme der Türkei in die EU bestehen würden) und sich zum willigen Diener des Auslands zu machen. Karakullukçu schrieb:

„Sie [der Vorstand der AABF] vereinen sich mit der [deutschen] Regierung, als hätten sie das gesamte alevitische Potential hinter sich, und sie vermitteln den Eindruck als könne dieser oder jener Vorteil dadurch erreicht werden. Aber ist es richtig die alevitische Gemeinschaft, ohne sie zu fragen auf diese Weise zum Material politischer und wirtschaftlicher Kalkulation gemäß der Wünsche Berlins zu machen, politischer und wirtschaftlicher Kalkulation ge
sogar auf Kosten der Interessen der Türkei?

Im Beitrag eines Lesers, der als Alevit identifiziert wurde, stand zu lesen, dass politische Kreise in Deutschland eine Kampagne gegen türkische Werte durchfuhrten und

„...einen Islam [erfinden], der dem Westen passt. Neue Identitatten wie ein deutsches Alevitentum werden erfunden. (...) Sie suchen nach Leuten, die einen Separatismus betreiben, der vom Westen gefordert wird. (...) Das anatolische Mosaik soll aufgebrochen werden, indem Organisationen auf der Grundlage aller möglichen Arten von ethnischen, religiösen und kulturellen Differenzen gefördert werden. Dic Organisationen, die auf dieser Basis gegrundet werden, werden vom Westen benutzt, um den Pfad der Entwicklung zu blockieren, der vom Nationalstaat unseres Geburtslandes gewählt wurde, und um es zu spalten. Wenn man das Ziel von all dem wissen möchte, genügt es, sich um unser Land herum umzuschauen: Der Plan, Jugoslawien zu teilen, steht vor unseren Augen." 20

19. Hürriyet, 5. Dezember 2000.

20 Hürriyet, 16. Januar 2001 
Der Leserbrief warf der Föderation weiter vor, von der Bundesregierung Geld für das Staatsbürgerschaftsprojekt genommen zu haben, um die Aleviten von ihrem Land" - damit ist die Türkei gemeint - zu trennen und eine europäische Identităt für sie bereitzustellen. Zusammengefasst werden die Vorwürfe, gegen die Interessen der Türkei zu arbeiten, in einer weiteren Kolumne von Karakullukçu. Er fragt dort rhetorisch:

„Wenn man die alevitische Gemeinschaft den Staaten Europas zum Geschenk macht, auf der Website der AABF scheußliche Völkermord-Anschuldigungen veröffentlicht und den Kurdistan-Phantasten in die Arme fält, wessen Interessen vertritt man dann?

\section{Assimilation und Separatismus}

Hürriyet beschuldigte die Föderation, sowohl Separatismus in der Türkei als auch Assimilation in Deutschland zu unterstutzen. Beide Vorwürfe sind auf der Basis des turkischen Nationsverständnisses in der Tat zwei Seiten derselben Medaille. Denn Assimilation bedeutet, dass die zentralen Werte des „Türkentums" und der Zugehörigkeit zur türkischen Nation aufgegeben werden, und dass damit Teile der Bevölkerung der türkischen Nation verloren gehen. Im essentialistischen Nationsverständnis des türkischen Diskurses ist das eigentlich gar nicht möglich. Wer ein Tüke ist, der bleibt auch ein Tüke, Veränderungen sind nicht vorgesehen. Es braucht schon "Verfuhrer", welche die Migranten auf Abwege bringen und sie bewusst von der Türkei "entfremden", damit „türkische": Migranten sich von "ihrer" Nation abwenden. Die Rolle eines solchen Verfuhrers weist Hürriyet der alevitischen Foderation in Deutschland zu. Damit steht die Zeitung nicht allein. Im Sommer 2001 warf Abdul Kadir Sezgin, ein ehemaliger hoher Beamter der Religionsbehörde, dem transnationalen alevitischen Dachverband „Alevi-Bektaşi Kuruluşları Birliği“", dem auch die $A A B F$ angehört, vor, einen eigenen Staat "Alevistan" gründen zu wollen. ${ }^{22} \mathrm{Er}$ bezeichnete die Aleviten als "die zweitgrößte separatistische Gefahr nach der PKK". 23

Nun wäre es falsch, die Angelegenheit so darzustellen, als sei der gesamte politische oder mediale Diskurs in der Turkei von derartigen Vorstellungen geprägt. Als der Separatismus-Vorwurf gegenüber den Aleviten erhoben wurde, da wurde auch vielfältiger Widerspruch geäußert. Im Sommer 2001 wurde sogar Ertuğ Karakullukçu als Herausgeber der Hürriyet-Europaausgabe abgelöst. Aber die Tatsache bleibt bestehen, dass solche Vorwürfe in der Türke

21 Hürriyet, 21. Dezember 2000. Die „scheußlichen Völkermord-Anschuldigungen" beziehen sich auf eine Internetseite zum Völkermord an den Armeniern, die ein Link zur Homepage der AABF beinhaltetete und daher von Hürriyet als Website der AABF identifiziert wurde. Tatsăchlich handelte es sich aber nicht um eine Seite der alevitischen Föderation.

22 Cumhuriyet, 20. August 2001.

23 Milliyet, 21. August 2001

176
Resonanz finden, und zwar nicht nur in der Presse. So war der SeparatismusVorwurf Gegenstand eines Prozesses in Ankara, in dem die Alevi-Bektaşi Kuruluşları Birliği im Herbst 2001 mit eben der Anschuldigung, Separatismus zu betreiben, verboten worden war. ${ }^{24}$

Der Separatismus-Vorwurf an die Föderation der Alevitengemeinden, wie er in Hürriyet geäußert wurde, fixiert die Zugehörigkeit der ,alevitischen Gemeinschaft" zur türkischen Nation. Denn Hürriyet konstruierte ja gerade einen Gegensatz zwischen der Föderation und den Mitgliedern ihres Vorstandes auf der einen sowie "der alevitischen Gemeinschaft" auf der anderen Seite. Nur die Vorstandsmitglieder der Föderation sind Hürriyet zufolge Separatisten und Feinde der Turkei. Mit dieser Haltung vertreten sie „die alevitische Gemeinschaft" ja gerade nicht. Die alevitische Gemeinschaft steht demnach im Gegensatz zur Föderation: Sie ist unverbrüchlicher Teil der türkischen Nation. Welche Haltung der alevitischen Einwanderer in Deutschland von Hürriyet gewünscht ist, ist in einer Kolumne vom 9. Dezember $2000 \mathrm{zu}$ lesen. Karakullukçu schrieb dort:

„Einer unserer geschätzten alevitischen Leser, der gestern Mittag anrief, sagte: ,Wir sind im Ausland, aber die Heimat ist in unserem Inneren.' Welch ein schönes Wort. (...) Er fügte hinzu: ,Wir haben niemals, nicht einmal in der Zeit der Massaker, daran gedacht, unser Vaterland und unsere Nation zu verraten." Natürlich war es so. Wer kann sich für die alevitische Gemeinschaft etwas anderes vorstellen? Wer kann auch nur ein Könchen Zweifel daran empfinden, dass unsere Menschen alevitischen Glaubens den grundsätzlichen Prinzipien der türkischen Republik, Atatürk und den Ideen Atatürks, dem Laizismus, der nationalen Einheit und dem Gefühl von Brüderlichkeit verbunden sind? So etwas gibt es nicht. So ist es nicht und so soll es nicht sein."

\section{Parallele Nationskonzepte und das Paradigma} kultureller Differenz

Aus deutscher Perspektive mag die Anschuldigung an die alevitische Föderation, Separatismus zu betreiben, alevitische Migranten in Deutschland der türkischen Nation zu entfremden, sie zu Deutschen machen zu wollen, und sich bei all dem als Handlanger der Bundesregierung zu betätigen, absurd erscheinen. Aber es ist aufschlussreich, die Parallelität von Nationsvorstellungen in Deutschland und in der Türkei in den Blick zu nehmen. Gemeinsam ist beiden Vorstellungen ein essentialistisches Konzept von nationaler Zugehörigkeit und Identität. In beiden Fällen wird die Zugehörigkeit als etwas durch Geburt und Abstammung Gegebenes betrachtet: Man ist Deutscher oder Türke - über Generationen hinweg, auch wenn man nicht im Territorium des jeweiligen Staates gelebt hat - und man kann es eigentlich auch nicht aufhören zu sein.

24 Im Oktober 2002 wurde das Verbot vom Kassationsgerichtshof in Ankara aufgehoben - allerdings war mit drei zu zwei Stimmen die Mehrheit fur die Aufhebung des Verbots im füfköpfigen Richtergremium sehr knapp. 
Ambivalenzen, Zwischenstadien, Hybridität oder mehrfache Zugehörigkeit (man denke an die Staatsbürgerschaftsdebatte in Deutschland) sind nicht vorgesehen. Hatte das türkische Nationsverständnis ursprünglich ein gewisses Element eines partizipatorischen Nationskonzeptes - denn Türke ist jeder, der Bürger der Türkei ist - so kamen im Bemühen, eine homogene Nation zu schaffen, ethnische Elemente und besonders die Sprache hinzu. ${ }^{25}$ Heute reicht es eben doch nicht allein aus, türkischer Staatsbürger zu sein. Man muss sich auch zur turkischen Kultur und Sprache bekennen.

Die Parallelităt der Nationsvorstellungen hat eine Komplementarität ihrer Wirkung auf Einwanderer aus der Tükei in Deutschland zur Folge. Aus der Perspektive des deutschen dominanten Diskurses bleiben eingewanderte Türken einfach Türken, Ausländer, Fremde. Die Möglichkeit, deutsch zu werden, ist vom Konzept her nicht vorgesehen, mögen individuelle Integrationsleistungen auch noch so groß sein. Die kulturelle Differenz zwischen Türken und Deutschen bleibt danach bestehen, die Einwanderer bleiben aus dem Konstrukt des „deutschen Volkes“ dauerhaft ausgeschlossen. ${ }^{26}$ Gleiches gilt umgekehrt für die Perspektive aus dem dominanten Nationaldiskurs der Türkei: Hier bleiben die Einwanderer in der turkischen Nation eingeschlossen, sie dürfen und können diese Nation nicht verlassen. Am Paradigma kultureller Differenz wird also sowohl vom Einwanderungsland, das keins sein will, als auch vom Herkunftsland, das nicht dauerhaft verlassen sein will, festgehalten. Die Einschließung des einen Nationaldiskurses doppelt die Ausschließung des anderen. Was dabei auf der Strecke bleibt, ist die Möglichkeit für Migranten, sowohl im Einwanderungsland als gleichberechtigte Bürger zu leben, als auch eine von ihnen selbst gewăhlten Form der Zugehörigkeit zum Herkunftsland zu praktizieren.

25 Zum Nationskonzept in der Turkei siehe Rumpf 1992. In den ersten Jahren der Republik wurde die Existenz einer kulturell eigenständigen Gruppe der Kurden in der Türkei nicht geleugnet - die Kurden galten damals als Kurden als Bürger der türkischen Republik. Dies änderte sich jedoch mit den ersten kurdischen der türkischen Republik. Dies änderte sich jedoch mit den ersten kurdischen
Aufständen: Sie wurden von der Regierung mit der Negation kurdischer Eigenständigkeit und dem Zwang zur Assimilation beantwortet.

26. Es bleibt abzuwarten, wie sich das neue Staatsangehörigkeitsrecht, das Einbürgerung erleichtert und unter bestimmten Umständen in Deutschland geborenen Kindern ausländischer Eltern die deutsche Staatsbürgerschaft verleiht, auf dieses gängige Vorstellung auswirken wird.

\section{Zitierte Literatur}

AABF (1998). Avrupa Alevi Birlikleri Federasyonu Programi. Köln.

Anderson, Benedict (1983): Imagined Communities, London: Verso.

Anderson, Benedict (1998): "Long-Distance Nationalism", In: ders., The Spectre of Comparison, London: Verso, S. 58-74.

Appadurai, Arjun (1989): „Disjuncture and Difference in the Global Cultural Economy", in: Public Culture 2: S. 1-24.

Brubaker, Rogers (1992): Citizenship and Nationhood in France and Germany, Cambridge, Mass.: Harvard University Press.

Ceyhun, Ozan (Hg.) (1999): Die türkischen Medien und die öffentliche Sicherheit in Europa. Die Grünen im Europäischen Parlament (Broschüre).

Cryns, Manfred/Özkan, Ayşe/Wedel, Heidi/Yarar-Zarif, Ferah (1991): Zum Integrationspotential der türkischen Tagespresse in der Bundesrepublik, Opladen: Leske und Budrich.

Dressler, Markus (1999): Die civil religion der Türkei. Kemalistische und alevitische Atatürk-Rezeptionen im Vergleich, Würzburg: Ergon.

Dressler, Marcus (2002): Die Alevitische Religion. Traditionslinien und Neubestimmung, Würzburg: Ergon.

Heinemann, Lars/Kamcil, Fuat (2000): „Unterhaltung, Absatzmärkte und die Vermittlung von Heimat. Die Rolle der Massenmedien in deutschtürkischen Räumen", in: Thomas Faist (Hg.), Transstaatliche Räume: Politik, Wirtschaft und Kultur in und zwischen Deutschland und der Türkei, Bielefeld: transcript, S. 113-153.

Karacabey, Makfi (1996): Türkische Tageszeitungen in der BRD. Rolle, Einfluß, Funktionen, Dissertation, Universität Frankfurt/Main.

Kehl-Bodrogi, Krisztina (1988): Die Klzllbaş/Aleviten. Untersuchungen über eine esoterische Glaubensgemeinschaft in Anatolien, Berlin: Klaus Schwarz Verlag.

Lewis, G. L. (1984): „Ataturk's Language Reform ans an Aspect of the Modernization in the Republic of Turkey", in: Jacob Landau (Hg.): Atatürk and the Modernization of Turkey, Boulder: Westview Press, S. 195-213.

Rumpf, Christian (1992): „Das Nationalismusprinzip in der türkischen Verfassung", in: Verfassung und Recht in Übersee 25, S. 407-445.

Schuler, Harald (1998): Die türkischen Parteien und ihre Mitglieder, Hamburg: Deutsches Orient Institut.

Shankland, David (1999): Islam and Society in Turkey, Huntingdon, Eothen Press.

Sorkefeld, Martin (2003): „Alevis in Germany and the Politics of Recognition", in: New Perspectives on Turkey 28-29. S. 133-161.

Sökefeld, Martin/Schwalgin, Susanne (2000): Institutions and their Agents in Diaspora. Transnational Communities Working Paper Scries, WPTC-2K11. Verfügbar im Internet: http://www.transcomm.ox.ac.uk

White, Paul J./Joost Jongerden (Hg.): Turkey's Alevi Enigma: A Comprehensive Overview, Leiden, Brill. 
Wimmer, Andreas (2002): Nationalist Exclusion and Ethnic Conflict: Shadows of Modernity, Cambridge: Cambridge University Press.

Yöruk, Zafer F. (1997): „Turkish Identity from Genesis to the Day of Judgement", in: Kathryn Dean (Hg.), Politics and the Ends of Identity, Aldershot: Ashgate, S.103-134.

Zitierte Tageszeitungen:

Cumhuriyet

die tageszeitung

Hüriyet

Milliyet

Özgür Politika 